\title{
Type 2 diabetes alters metabolic and transcriptional signatures of glucose and amino acid metabolism during exercise and recovery
}

\author{
Jakob S. Hansen ${ }^{1,2} \cdot$ Xinjie Zhao $^{3} \cdot$ Martin Irmler $^{4} \cdot$ Xinyu Liu $^{3} \cdot$ Miriam Hoene $^{5}$. \\ Mika Scheler ${ }^{4,6}$ - Yanjie $\mathrm{Li}^{3}$ • Johannes Beckers ${ }^{4,6,7}$ - Martin Hrabě de Angelis ${ }^{4,6,7}$. \\ Hans-Ulrich Häring ${ }^{5,6,8}$ • Bente K. Pedersen ${ }^{1}$. Rainer Lehmann ${ }^{5,6,8}$ • Guowang $\mathrm{Xu}^{3}$ • \\ Peter Plomgaard $^{1,2} \cdot$ Cora Weigert ${ }^{5,6,8}$
}

Received: 21 November 2014 / Accepted: 13 March 2015 /Published online: 12 June 2015

(C) Springer-Verlag Berlin Heidelberg 2015

\begin{abstract}
Aims/hypothesis The therapeutic benefit of physical activity to prevent and treat type 2 diabetes is commonly accepted. However, the impact of the disease on the acute metabolic response is less clear. To this end, we investigated the effect of type 2 diabetes on exercise-induced plasma metabolite changes and the muscular transcriptional response using a complementary metabolomics/transcriptomics approach.

Methods We analysed 139 plasma metabolites and hormones at nine time points, and whole genome expression in skeletal muscle at three time points, during a $60 \mathrm{~min}$ bicycle ergometer exercise and a 180 min recovery phase in type 2 diabetic patients and healthy controls matched for age, percentage body fat and maximal oxygen consumption $\left(\dot{V} \mathrm{O}_{2 \max }\right)$.
\end{abstract}

Electronic supplementary material The online version of this article (doi:10.1007/s00125-015-3584-x) contains peer-reviewed but unedited supplementary material, which is available to authorised users.

Guowang Xu

xugw@dicp.ac.cn

Peter Plomgaard

plomgaard@dadlnet.dk

Cora Weigert

cora.weigert@med.uni-tuebingen.de

1 Centre of Inflammation and Metabolism, Centre for Physical Activity Research, Department of Infectious Diseases, Rigshospitalet, Blegdamsvej 9, 2100 Copenhagen, Denmark

2 Department of Clinical Biochemistry, Rigshospitalet, Copenhagen, Denmark

3 Key Laboratory of Separation Science for Analytical Chemistry, Dalian Institute of Chemical Physics, Chinese Academy of Sciences, 457 Zhongshan Road, Dalian 116023, China
Results Pathway analysis of differentially regulated genes upon exercise revealed upregulation of regulators of GLUT4 (SLC2A4RG, FLOT1, EXOC7, RAB13, RABGAP1 and $C B L B)$, glycolysis (HK2, PFKFB1, PFKFB3, PFKM, FBP2 and $L D H A)$ and insulin signal mediators in diabetic participants compared with controls. Notably, diabetic participants had normalised rates of lactate and insulin levels, and of glucose appearance and disappearance, after exercise. They also showed an exercise-induced compensatory regulation of genes involved in biosynthesis and metabolism of amino acids (PSPH, GATM, NOS1 and GLDC), which responded to differences in the amino acid profile (consistently lower plasma levels of glycine, cysteine and arginine). Markers of fat oxidation (acylcarnitines) and lipolysis (glycerol) did not indicate

4 Institute of Experimental Genetics, Helmholtz Zentrum München, German Research Center for Environmental Health $(\mathrm{GmbH})$, Neuherberg, Germany

5 Division of Pathobiochemistry and Clinical Chemistry, Department of Internal Medicine, University of Tübingen, Otfried-Müller-Str. 10, 72076 Tübingen, Germany

6 German Center for Diabetes Research (DZD), Germany, http://www.dzd-ev.de/en

7 Chair of Experimental Genetics, Center of Life and Food Sciences Weihenstephan, Technische Universität München, FreisingWeihenstephan, Germany

8 Institute for Diabetes Research and Metabolic Diseases of the Helmholtz Zentrum München, University of Tübingen, Tübingen, Germany 
impaired metabolic flexibility during exercise in diabetic participants.

Conclusions/interpretation Type 2 diabetic individuals showed specific exercise-regulated gene expression. These data provide novel insight into potential mechanisms to ameliorate the disturbed glucose and amino acid metabolism associated with type 2 diabetes.

Keywords Basic science $\cdot$ Exercise $\cdot$ Human . Metabolomics $\cdot$ Microarray $\cdot$ Pathophysiology/metabolism

$\begin{array}{ll}\text { Abbreviations } \\ \text { HK2 } & \text { Hexokinase } 2 \\ \text { KEGG } & \text { Kyoto Encyclopedia of Genes and Genomes } \\ \text { NGT } & \text { Normal glucose tolerant } \\ \text { PFKFB } & \begin{array}{l}\text { 6-Phosphofructo-2-kinase/fructose-2,6- } \\ \text { bisphosphatase }\end{array} \\ \text { PFKM } & \begin{array}{l}\text { Phosphofructokinase, muscle } \\ \text { PGC1 } \alpha\end{array} \\ \begin{array}{ll}\text { Peroxisome proliferator-activated receptor gamma } \\ \text { coactivator 1 } \alpha\end{array} \\ R_{\mathrm{a}} & \begin{array}{l}\text { Glucose rate of appearance } \\ R_{\mathrm{d}}\end{array} \\ \dot{V} \mathrm{O}_{2 \max } & \text { Glucose rate of disappearance } \\ \end{array}$

\section{Introduction}

Chronic metabolic disorders such as obesity, insulin resistance and type 2 diabetes are characterised by an insufficient and disturbed capacity to store and oxidise glucose and lipids $[1,2]$ and are exacerbated by physical inactivity [3]. Conversely, exercise has been shown to improve glycaemic control, whole-body fat oxidation and insulin sensitivity and is recommended for both the prevention and treatment of type 2 diabetes [4-6]. If regularly performed, physical exercise initiates transcriptional and (post)translational mechanisms that increase the capacity and efficiency of the muscle to utilise fuels [7]. Great effort is being undertaken to provide tailored, motivating exercise intervention programmes [8]. However, despite numerous human and rodent exercise studies, the complex nature of the mechanisms that lead to beneficial metabolic changes is incompletely understood. In particular, little is known whether the existence of diabetes has consequences for the acute metabolic response to exercise and the therapeutic effect of exercise training.

Studies before the 'omics' era focused on differences in substrate utilisation and metabolism during exercise in diabetic patients and controls using tracer infusion techniques [9-11]. Emerging metabolite profiling technologies have now enabled comprehensive analyses of metabolic intermediates in body fluids and allow detailed investigation of complex plasma metabolite changes during exercise and recovery
$[12,13]$. In addition, whole genome expression analyses have elucidated the global transcriptional response of human muscle to exercise [14, 15], but the impact of diabetes on exerciseregulated genes is unclear.

We therefore aimed to deepen our understanding of the acute molecular responses of type 2 diabetic patients to physical activity by carrying out a detailed investigation of plasma metabolite profiles combined with the transcriptional signatures of the exercising muscle. To this end, we used a targeted liquid chromatography-mass spectrometry-based metabolomics platform [16] to study the plasma metabolome of normal glucose tolerant (NGT) and diabetic individuals in serial blood samples obtained during a $60 \mathrm{~min}$ bicycle ergometer exercise and a $180 \mathrm{~min}$ recovery phase. To compare individuals with a similar fitness level, NGT and diabetic participants were matched not only for age and percentage body fat but also for maximal oxygen consumption $\left(\dot{V} \mathrm{O}_{2 \max }\right)$. $\left[\mathrm{U}_{-}{ }^{13} \mathrm{C}\right]$ Glucose was infused intravenously to assess the glucose rate of appearance $\left(R_{\mathrm{a}}\right)$ and disappearance $\left(R_{\mathrm{d}}\right)$. Finally, we integrated the metabolomics data with whole genome expression data of skeletal muscle biopsies obtained from the same individuals at three time points: before and after exercise and at the end of the recovery phase.

\section{Methods}

Participants The study comprised eight male patients with type 2 diabetes and eight healthy male volunteers (NGT group). Diabetic participants were receiving glucoselowering treatment with metformin $(n=5)$, metformin and glimepiride $(n=1)$ or diet, as glucose-lowering treatment, together with simvastatin $(n=4)$. The oral glucose-lowering treatment was paused 1 week prior to the exercise experiment, but treatment with simvastatin was continued. Of the eight patients originally included in the diabetic group, one was excluded in all final analyses due to very high fasting glucose concentrations $(>13 \mathrm{mmol} / \mathrm{l})$ on the day of the experiment.

The study was approved by the local ethics committee and conducted in accordance with the Declaration of Helsinki. All participants gave written informed consent before the study commenced. Pre-experimental assessments are shown in the electronic supplementary material (ESM Methods).

Exercise experiment All participants were instructed to refrain from strenuous physical activity $24 \mathrm{~h}$ prior to the day of the experiment and not to change their diet. On the day of the experiment, participants reported to the laboratory at 07:30 hours after an overnight fast from 22:00 hours the preceding day. A catheter was placed in an antecubital vein on each arm and baseline blood samples were drawn. After the baseline blood sampling, a prime bolus of $17.6 \mu \mathrm{mol} / \mathrm{kg}$ $\left[\mathrm{U}-{ }^{13} \mathrm{C}\right]$ glucose was injected intravenously. This was followed 
by a continuous infusion of $\left[\mathrm{U}^{13} \mathrm{C}\right] \mathrm{glucose}$ (rate: $0.4 \mu \mathrm{mol} \mathrm{kg}{ }^{-1} \mathrm{~min}^{-1}$ at rest and $0.8 \mu \mathrm{mol} \mathrm{kg}{ }^{-1} \mathrm{~min}^{-1}$ during the trial). [U- $\left.{ }^{13} \mathrm{C}\right]$ Palmitate was infused (rate: $0.015 \mu \mathrm{mol} \mathrm{kg}^{-1} \mathrm{~min}^{-1}$ at rest and $0.03 \mu \mathrm{mol} \mathrm{kg}^{-1} \mathrm{~min}^{-1}$ during the trial) to measure acylcarnitine enrichment. After $150 \mathrm{~min}$ to allow the tracers to reach steady state, participants completed a $60 \mathrm{~min}$ bicycle ergometer exercise at approximately $50 \%$ of their individual $\dot{V} \mathrm{O}_{2 \max }$. Blood samples were drawn at time points 5, 10, 30 and 60 min during exercise and at 90,120, 180 and 240 min during recovery. Blood was collected in EDTA-coated containers and immediately spun into plasma at $4^{\circ} \mathrm{C}$. Plasma samples were stored at $-80^{\circ} \mathrm{C}$ until analysis. The participants fasted until the last blood sample was obtained, but had free access to water. Skeletal muscle biopsies were obtained immediately prior to the exercise bout, immediately post exercise and after $180 \mathrm{~min}$ of recovery.

Plasma analysis Clinical chemical routine variables were measured using an ADVIA 1650 clinical chemical analyser (Siemens Healthcare Diagnostics, Fernwald, Germany); insulin, cortisol and growth hormone were analysed using an ADVIA Centaur immunoassay system (Siemens Healthcare Diagnostics). Glucagon was measured by RIA (Millipore, Bedford, MA, USA). Plasma glucose isotope enrichment, tracer-to-tracee ratio (TTR), was measured by liquid chromatography-mass spectrometry after derivatisation, as previously described [17]. Glucose $R_{\mathrm{a}}$ and $R_{\mathrm{d}}$ were calculated using a steady-state model where [18]:

$R_{\mathrm{a}}=R_{\mathrm{d}}=$ isotope infusion rate $\left(\mu \mathrm{mol} \mathrm{kg} \mathrm{kgi}^{-1}\right) / \mathrm{TTR}$.

Metabolic profiling The samples were quantitatively analysed using an ultra-HPLC 1290 Infinity system (Agilent, Santa Clara, CA, USA) coupled to a 6400 Triple Quad mass spectrometry system (Agilent) (ESM Methods). The following were measured: 37 amino acids and amines, 37 fatty acids, 42 acylcarnitines and 14 lysophosphatidylcholines.

RNA isolation and microarray analysis Snap-frozen human muscle biopsies were homogenised using a TissueLyser II (Qiagen, Hilden, Germany). Amplified cDNA was hybridised on Affymetrix Human Gene 1.0 ST arrays (Santa Clara, CA, USA) (ESM Methods). One NGT participant did not meet the criteria for good-quality microarray data and was excluded from the analysis.

Statistical analysis Statistical analyses of microarray data were performed using the statistical programming environment R implemented in CARMAweb [19]. Gene-wise testing for differential expression of time course analyses was carried out using the paired limma $t$ test in combination with the Benjamini-Hochberg multiple testing correction (false discovery rate $<10 \%$, average expression in at least one group $>8$ ). Differential gene regulation between diabetic and NGT samples at the indicated time points was done by applying the limma $t$ test on $\log _{2}$ ratios (average expression $>8$ ). Data were analysed through the use of Qiagen's Ingenuity Pathway Analysis (www.qiagen.com/ingenuity). Enriched Kyoto Encyclopedia of Genes and Genomes (KEGG) pathways associated with differences in gene expression and metabolite levels were identified using InCroMAP [20]. Two-way ANOVA analysis was used to assess the effect of time and groups on measurements over time. If there was a statistically significant difference over time, one-way ANOVA was used to assess the effect of time in each group. To assess differences between groups on baseline parameters, an unpaired $t$-test was used. A $p$ value $<0.05$ was considered statistically significant. All analyses were performed using SAS software, version 9.1 (SAS Institute, Cary, NC, USA).

\section{Results}

Baseline characteristics of participants Baseline characteristics of the participants are presented in Table 1. In summary, the participants were matched for age, percentage body fat, and $\dot{V} \mathrm{O}_{2 \max }$. Compared with the NGT group, the diabetic group had higher fasting and $2 \mathrm{~h}$ glucose concentrations during the OGTT and higher $\mathrm{HbA}_{1 \mathrm{c}}$ levels. Diabetic participants met the diagnostic criteria for type 2 diabetes of $2 \mathrm{~h}$ glucose levels $>11.1 \mathrm{mmol} / \mathrm{l}$.

Glucose $R_{\mathbf{a}} / R_{\mathbf{d}}$ and hormonal responses during the trial All participants completed a 60 min bicycle ergometer exercise at $50 \%$ of their individual $\dot{V} \mathrm{O}_{2 \max }$. The glucose concentration did not change during the experiment in either group; diabetic participants had higher glucose levels (Fig. 1a). Lactate levels were higher before exercise in the diabetic group $(1.06 \pm 0.11$ vs $0.70 \pm 0.06 \mathrm{mmol} / \mathrm{l}, p=0.014)$ and reached a higher plateau immediately after the start of exercise (Fig. 1b). Lactate returned to comparable, lower levels after $30 \mathrm{~min}$ of recovery in both groups. Diabetic participants had higher glucose $R_{\mathrm{a}} / R_{\mathrm{d}}$ at baseline (Fig. 1c). During exercise, glucose $R_{\mathrm{a}} / R_{\mathrm{d}}$ increased markedly to a similar level in both groups and decreased to comparable values in the recovery phase, indicating normalisation in the diabetic group. Plasma glycerol constantly increased during exercise in both groups and declined rapidly afterwards, indicating induction and inhibition of lipolysis (Fig. 1d). However, while similar before and during exercise, glycerol levels remained higher in the diabetic group after exercise. Insulin levels were higher before and during exercise in diabetic participants but did not differ significantly in the recovery phase (Fig. 1e). In both groups the highest insulin concentrations were seen at 90 min, the first time point in the recovery phase. Growth hormone and cortisol increased in response to exercise in both 
Table 1 Baseline characteristics of NGT and type 2 diabetic participants

\begin{tabular}{|c|c|c|c|}
\hline Variable & $\operatorname{NGT}(n=8)$ & Type 2 diabetes $(n=7)$ & $p$ value \\
\hline \multicolumn{4}{|l|}{ Basic characteristics } \\
\hline Age, years & $56.1 \pm 2.2$ & $57.3 \pm 1.7$ & 0.684 \\
\hline Height, $\mathrm{cm}$ & $182.4 \pm 1.8$ & $179.4 \pm 2.4$ & 0.334 \\
\hline Weight, kg & $91.2 \pm 3.8$ & $78.9 \pm 2.9$ & 0.023 \\
\hline BMI, $\mathrm{kg} / \mathrm{m}^{2}$ & $27.4 \pm 1.1$ & $24.6 \pm 1.0$ & 0.082 \\
\hline Body fat, $\%$ & $24.1 \pm 2.3$ & $26.5 \pm 1.8$ & 0.435 \\
\hline Heart rate, bpm & $60.9 \pm 4.9$ & $66.3 \pm 5.1$ & 0.458 \\
\hline Systolic blood pressure, $\mathrm{mmHg}$ & $141.5 \pm 6.6$ & $139.6 \pm 3.2$ & 0.797 \\
\hline Diastolic blood pressure, $\mathrm{mmHg}$ & $92.0 \pm 2.9$ & $88.4 \pm 3.2$ & 0.425 \\
\hline \multicolumn{4}{|l|}{ Fitness } \\
\hline$\dot{V} \mathrm{O}_{2 \max }, \mathrm{ml} \mathrm{kg}^{-1} \min ^{-1}$ & $37.0 \pm 2.0$ & $32.4 \pm 1.8$ & 0.112 \\
\hline \multicolumn{4}{|l|}{ Glucose variables } \\
\hline Fasting glucose, $\mathrm{mmol} / \mathrm{l}$ & $5.1 \pm 0.2$ & $7.1 \pm 0.5$ & 0.005 \\
\hline Fasting insulin, pmol/1 & $38.3 \pm 8.6$ & $76.0 \pm 17.9$ & 0.094 \\
\hline Fasting C-peptide, pmol/1 & $629.3 \pm 45.9$ & $967.7 \pm 127.4$ & 0.039 \\
\hline $2 \mathrm{~h}$ glucose, $\mathrm{mmol} / \mathrm{l}$ & $5.0 \pm 0.6$ & $15.2 \pm 1.2$ & $<0.001$ \\
\hline $\mathrm{HbA}_{1 \mathrm{c}}, \%$ & $5.5 \pm 0.1$ & $6.4 \pm 0.2$ & 0.004 \\
\hline $\mathrm{HbA}_{1 \mathrm{c}}, \mathrm{mmol} / \mathrm{l}$ & $37 \pm 1.1$ & $46 \pm 2.2$ & \\
\hline \multicolumn{4}{|l|}{ Biochemical variables } \\
\hline Haemoglobin, mmol/1 & $8.9 \pm 0.2$ & $9.0 \pm 0.1$ & 0.544 \\
\hline Leucocytes, $\times 10^{9} / 1$ & $5.5 \pm 0.5$ & $5.8 \pm 0.5$ & 0.627 \\
\hline Creatinine, $\mu \mathrm{mol} / 1$ & $79.9 \pm 5.4$ & $66.0 \pm 2.2$ & 0.041 \\
\hline Alanine aminotransferase, $\mu \mathrm{kat} / \mathrm{l}$ & $0.42 \pm 0.04$ & $0.53 \pm 0.05$ & 0.076 \\
\hline Factor II, VII, X, $\mathrm{AU}^{\mathrm{a}}$ & $0.9 \pm 0.1$ & $0.9 \pm 0.1$ & 0.738 \\
\hline Total cholesterol, mmol/1 & $5.1 \pm 0.2$ & $4.4 \pm 0.3$ & 0.107 \\
\hline HDL-cholesterol, mmol/1 & $1.5 \pm 0.1$ & $1.7 \pm 0.1$ & 0.227 \\
\hline LDL-cholesterol, mmol/1 & $3.0 \pm 0.2$ & $2.3 \pm 0.2$ & 0.050 \\
\hline Triacylglycerol, mmol/1 & $0.8 \pm 0.1$ & $1.0 \pm 0.2$ & 0.375 \\
\hline C-reactive protein, nmol/1 & $13.3 \pm 3.8$ & $10.5 \pm 1.0$ & 0.577 \\
\hline
\end{tabular}

Data are mean \pm SEM

$p$ values were calculated using unpaired $t$ test

${ }^{a}$ Arbitrary units, expressed as a proportion of 1.0 groups (Fig. 1f, g). Growth hormone levels were lower in the diabetic group after exercise. Glucagon levels did not differ between groups during the trial and modestly increased during exercise, reaching significance only in controls (Fig. 1h).

\section{Effect of type 2 diabetes on metabolite changes during} exercise and recovery Metabolic profiling revealed that 62 plasma metabolites significantly changed during the trial when all participants were analysed together (ESM Fig. 1; $p<0.05$, one-way ANOVA). The metabolites that responded to exercise and differed in their concentration between the diabetic group and the NGT group are shown in ESM Table 1.

Alanine was increased after 30 and $60 \mathrm{~min}$ of exercise in the diabetic group compared with the NGT group (Fig. 2a; ESM Table 1), with a clear correlation between the higher lactate and higher alanine concentrations at 5, 10, 30, 60 and
$90 \mathrm{~min}$ of the trial, but not at baseline or at 120 and $180 \mathrm{~min}$ (Fig. 2b; ESM Table 2). Glutamate levels were higher in diabetic participants during exercise (Fig. 2c), with significantly higher baseline values $(15.3 \pm 1.2$ vs $9.6 \pm 1.3 \mu \mathrm{mol} / 1, p=$ 0.005). The concentrations of the amino acids arginine, asparagine, cysteine, glutamine, glycine and threonine were lower in the diabetic group and did not change during the trial (ESM Table 3 and ESM Fig. 2). $\beta$ Aminoisobutyric acid was not regulated upon exercise and there was a tendency towards lower levels in diabetic participants ( $p=0.08$; ESM Fig. 2).

The diabetic state had a strong effect on the regulation during recovery of saturated and monounsaturated fatty acids with 14-19 carbon atoms ( $p<0.05$ for time $\times$ group effect). In diabetic participants, fatty acids showed a clear increase after $90 \mathrm{~min}$ and remained at higher concentrations after 120 and 

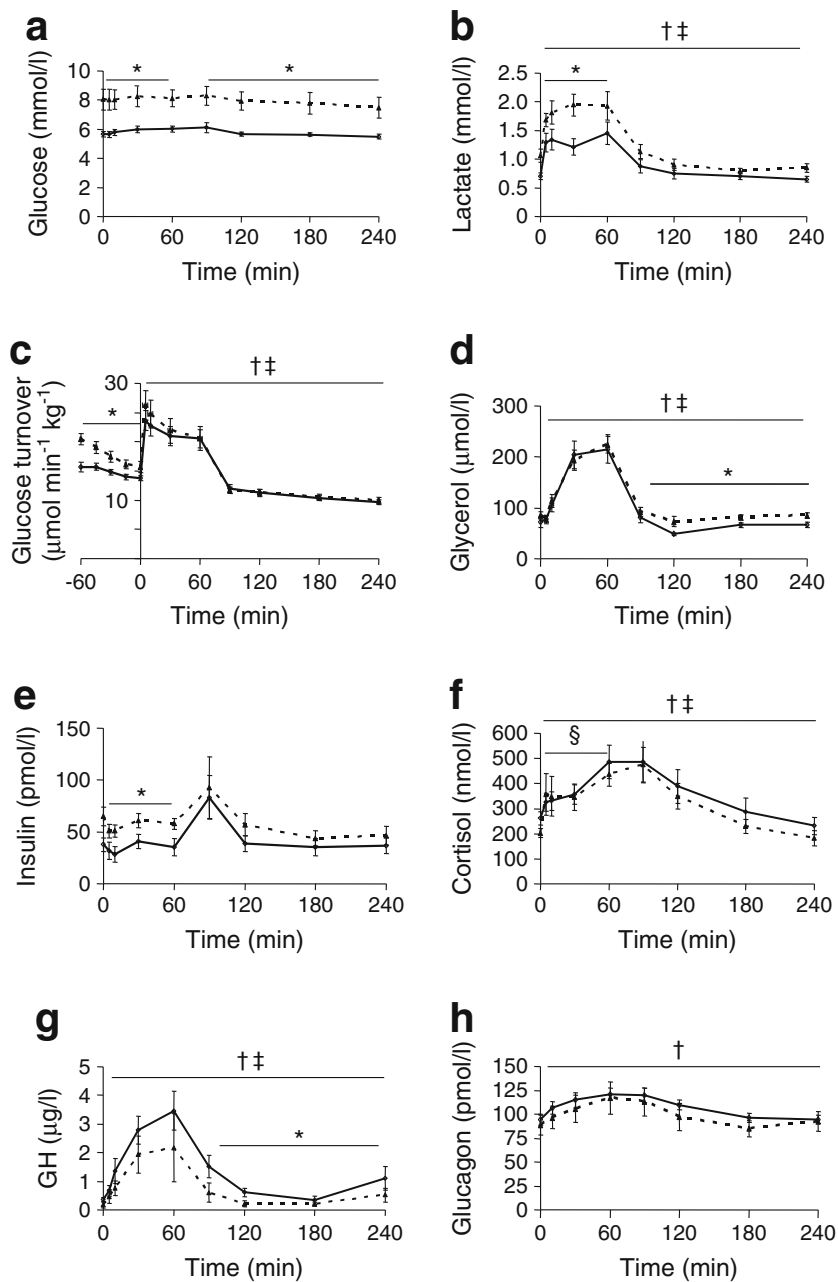

Fig. 1 Glucose $R_{\mathrm{a}} / R_{\mathrm{d}}$ and hormonal responses during the trial. Plasma concentrations (mean $\pm \mathrm{SEM}$ ) of glucose (a), lactate $(\mathbf{b})$, glucose $R_{\mathrm{a}} / R_{\mathrm{d}}(\mathbf{c})$, glycerol (d), insulin (e), cortisol (f), growth hormone (GH) (g) and glucagon (h) in NGT (solid lines) and type 2 diabetic participants (dotted lines) during the trial; ${ }^{*} p<0.05$ for difference between the two groups tested by two-way ANOVA; ${ }^{\dagger}$ significant effect over time in the NGT group as indicated in the figure ( $p<0.05$ tested by one-way ANOVA); ${ }^{*}$ significant effect over time in the type 2 diabetic group as indicated in the figure $\left(p<0.05\right.$ tested by one-way ANOVA); ${ }^{\S} p<0.05$ for time $\times$ group effect tested by two-way ANOVA

$180 \mathrm{~min}$, as exemplarily shown for fatty acids C14, C15, C16 and $\mathrm{C} 18$ (Fig. 2d-g).

Acylcarnitines showed a strikingly similar response in the two groups during the trial, particularly during the exercise phase (Fig. $2 \mathrm{~h}-\mathrm{j}$ ). Medium chain acylcarnitines were strongly increased in the two groups and reached a similar peak after $60 \mathrm{~min}$ (Fig. 2h). The higher concentrations of acylcarnitine C14 in diabetic participants may mainly reflect the higher availability of the corresponding fatty acid C14 (Fig. 2i). Furthermore, no difference was seen in the regulation of acetylcarnitine $\mathrm{C} 2$, which not only reflects fatty acid oxidation but also reflects oxidative glucose metabolism (Fig. 2j). The plasma level of free carnitine did not differ between the two groups (concentration before exercise: $44.3 \pm 6.6 \mu \mathrm{mol} / 1$ in controls vs $45.6 \pm 5.0 \mu \mathrm{mol} / 1$ in diabetic participants) and did not change during the trial (Fig. $2 \mathrm{k}$ ). The ratio of $\mathrm{C} 2$ and $\mathrm{C} 3$ acylcarnitine to carnitine as an indicator of $\beta$-oxidation was increased during exercise and peaked after $90 \mathrm{~min}$, with no difference between NGT and diabetic participants (data not shown).

Lysophosphatidylcholines differed in their concentration in the diabetic state but were not regulated by exercise (ESM Table 3).

Exercise-regulated genes in skeletal muscle common to type 2 diabetic and control participants Assessment of skeletal muscle gene regulation revealed sets of commonly regulated genes upon exercise in the two groups. We observed 29 significantly regulated probe sets immediately after exercise (60 $\mathrm{min}$ ) and 21 after recovery (240 $\mathrm{min}$ ) (false discovery rate $<10 \%$; fold change $>1.5$; ESM Fig. 3 ). Genes encoding key regulators of the metabolic adaptation to exercise, PPARGC1A (encoding peroxisome proliferator-activated receptor gamma coactivator $1 \alpha[\mathrm{PGC} 1 \alpha]$ ), NR4A3 (encoding the nuclear receptor subfamily 4, group A3), VEGFA (encoding vascular endothelial growth factor) and $E G R I$ (encoding early growth response 1) were upregulated in the two groups. Stress response genes were also induced to a similar level, namely HMOX1 (encoding haem oxygenase) and HSPH1, DNAJB1 and DNAJA1 (encoding heat shock proteins).

Different transcriptional response of skeletal muscle genes in the type 2 diabetic group and the relation to metabolic pathways Exercise-dependent differential gene regulation between muscle biopsies obtained from NGT and diabetic participants was found for 189 and 229 probe sets after 60 or $240 \mathrm{~min}$, respectively ( $p<0.01$, ratio of fold change $>1.2$; data not shown). Significantly enriched KEGG pathways with differentially regulated probe sets and metabolites or differentially regulated probe sets only are shown in ESM Table 4 $(p<0.05)$. The enrichment for the insulin signalling pathway is illustrated by exercise-induced upregulation of insulin signalling components (ESM Fig. 4 and ESM Table 5) and of regulators of GLUT4 (encoded by SLC2A4) translocation and expression (Fig. 3; ESM Tables 5 and 6). Further enrichment of genes was found for the regulation of glycolysis (Fig. 3; ESM Table 6). Expression of $H K 2$ (encoding hexokinase 2), $P F K F B 1$ and PFKFB3 (encoding 6-phosphofructo-2-kinase/ fructose-2,6-bisphosphatase), $P F K M$ (encoding phosphofructokinase, muscle), FBP2 (encoding fructose-1,6bisphosphatase) and $L D H A$ (encoding lactate dehydrogenase) was induced by exercise in diabetic participants compared with controls. Together with the increased plasma levels of lactate and alanine, this reflects adaptation to higher nonoxidative glucose metabolism during exercise in the diabetic group. Enrichment was also found in the biosynthesis and 

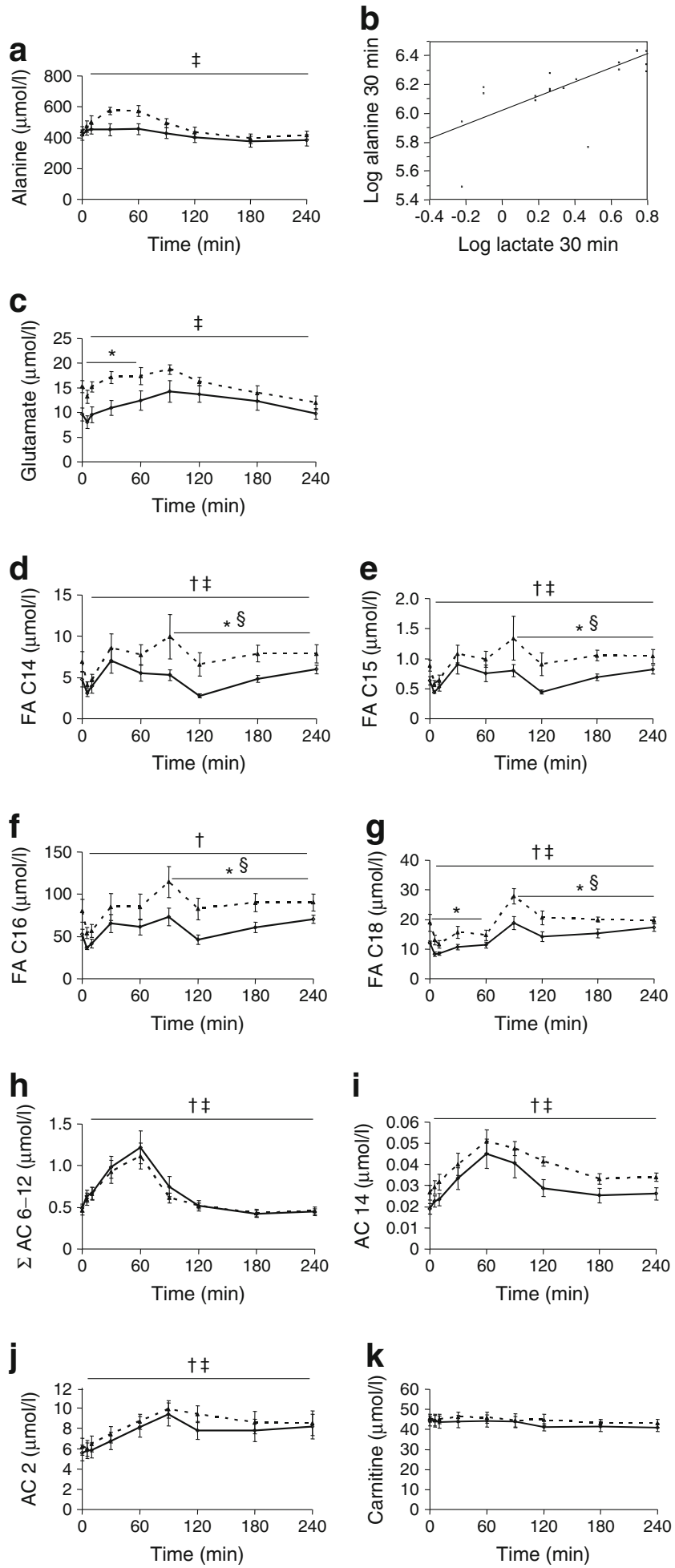

metabolism of amino acid pathways (Fig. 4; ESM Tables 4 and 6). The differential expression of GATM (encoding glycine amidinotransferase), GLDC (encoding glycine dehydrogenase, decarboxylating), GLUL (encoding glutamateammonia ligase), GPT1 (encoding glutamic-pyruvate transaminases), NOS1 (encoding nitric oxide synthase) and $P S P H$
Fig. 2 Amino acids and fatty acids (FA) showing higher plasma concentrations in type 2 diabetes during exercise or recovery. (a-c) Amino acids with changes in plasma concentrations during the trial that show differences between NGT (solid lines) and type 2 diabetic participants (dotted lines). Time curves represent the plasma concentrations of alanine (a) and glutamate (c) of both groups. (b) Correlation of log-transformed $\left(\log _{e} x\right)$ lactate and alanine plasma concentrations at $30 \mathrm{~min}\left(p=0.0003, r^{2}=0.64\right)$. $(\mathbf{d}-\mathbf{g}) \mathrm{FA} \mathrm{C} 14, \mathrm{C} 15$, $\mathrm{C} 16$ and $\mathrm{C} 18$ were differentially regulated between NGT (solid lines) and type 2 diabetic participants (dotted lines) in the recovery phase. (hk) Sum of all acylcarnitines (AC) C6-C12 ( $\Sigma$ AC 6-12), AC 14, AC 2 and carnitine in NGT (solid lines) and type 2 diabetic participants (dotted lines). Values in $(\mathbf{a}-\mathbf{k})$ are shown as mean \pm SEM. ${ }^{*} p<0.05$ for difference between the two groups tested by two-way ANOVA; ${ }^{\dagger}$ significant effect over time in the NGT group as indicated in the figure $(p<0.05$ tested by one-way ANOVA); ${ }^{\star}$ significant effect over time in the type 2 diabetic group as indicated in the figure ( $p<0.05$ tested by one-way ANOVA); ${ }_{p}<0.05$ for time $\times$ group effect tested by two-way ANOVA

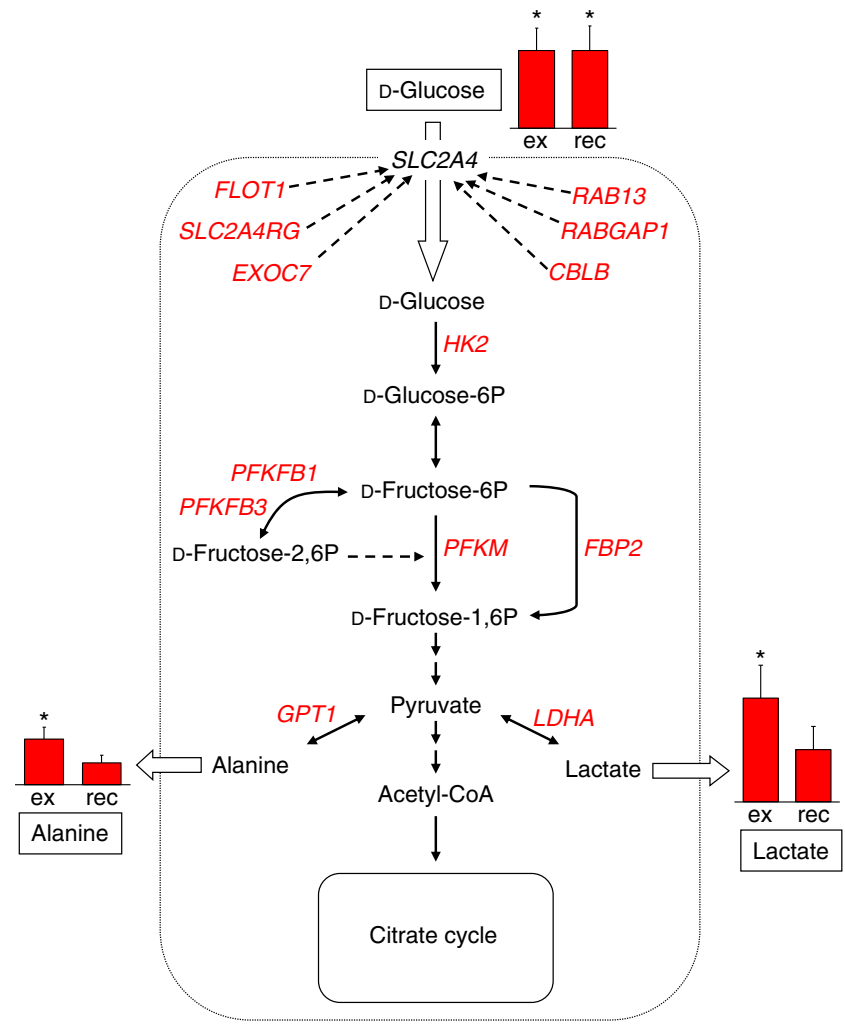

Fig. 3 Differences in metabolite concentrations and gene expression reflected higher glucose degradation in type 2 diabetes during the trial. The figure illustrates glucose uptake and metabolism in skeletal muscle. Metabolites analysed in our study are included in text boxes. Histograms show the ratio of the $\mathrm{AUC}_{0-60}$ min (exercise [ex]) or of $\mathrm{AUC}_{60-240}$ min (recovery [rec]) of type 2 diabetic vs NGT participants of significantly different metabolites according to ESM Tables 1 and 3 (mean \pm SEM; ${ }^{*} p<0.05$ type 2 diabetes vs NGT). Differentially regulated genes after exercise (60 or $240 \mathrm{~min}$; type 2 diabetes vs NGT, ratio of fold change $>1.2, p<0.05$; ESM Table 6 ) are shown in italic capitals: red for upregulation. Regulators of $S L C 2 A 4$ expression or translocation are indicated by dashed arrows. D-Fructose-2,6P regulates PFKM activity. Dotted line indicates plasma membrane 


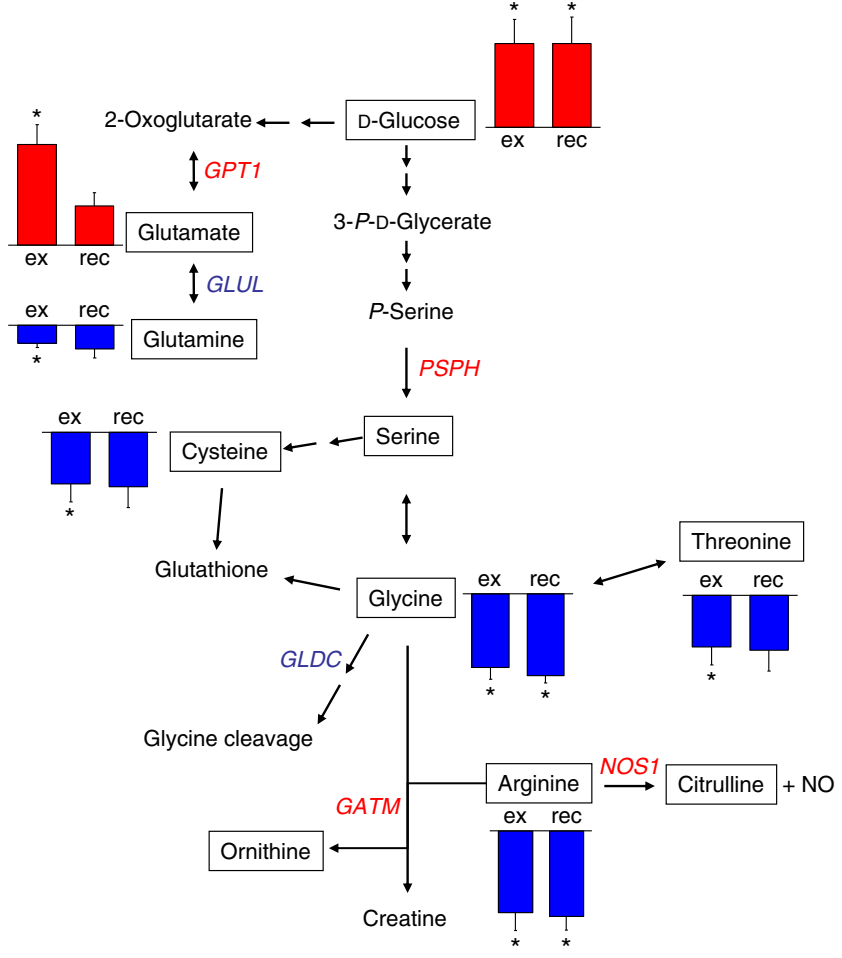

Fig. 4 Different transcriptional responses of diabetic skeletal muscle and the relation to differences in plasma amino acid concentrations. The figure illustrates biosynthesis and metabolism of amino acids. Metabolites analysed in our study are included in text boxes. Histograms show the ratio of the $\mathrm{AUC}_{0-60}$ min (exercise [ex]) or of $\mathrm{AUC}_{60-240}$ min (recovery [rec]) of type 2 diabetic vs NGT participants of significantly different metabolites according to ESM Tables 1 and 3 (mean \pm SEM; ${ }^{*} p<0.05$ type 2 diabetes vs NGT). Concentrations of serine, ornithine and citrulline were not different between the two groups. Differentially regulated genes after exercise ( 60 or $240 \mathrm{~min}$; type 2 diabetes vs NGT, ratio of fold change $>1.2, p<0.05$; ESM Table 6 ) are shown in italic capitals: red for upregulation, blue for downregulation; $\mathrm{NO}$, nitric oxide

(encoding phosphoserine phosphatase) is clearly associated with the different plasma levels of glutamate, glycine, cysteine, threonine and arginine.

\section{Discussion}

In this study we showed that type 2 diabetic patients have higher non-oxidative glucose metabolism during exercise, evidenced by higher plasma lactate and alanine levels. After exercise, diabetic participants had normalised rates of glucose $R_{\mathrm{a}}$ and $R_{\mathrm{d}}$, lactate and insulin levels. The exercise-induced increase in fatty acid oxidation and lipolysis was similar in diabetic and NGT participants. Upon exercise, specific transcriptional adaptations were found in the diabetic skeletal muscle that could be a consequence of the disturbed glucose and amino acid metabolism in the diabetic state.

The exercise-induced increased expression of regulators of glucose transport and metabolism, as well as insulin signal mediators, found in the diabetic participants may be involved in long-term improvement. The glycolytic enzymes HK2 and PFKM and the bidirectional regulators of glycolysis PFKFB1 and PFKFB3 were upregulated after exercise in the diabetic participants. Notably, enhanced expression of $P F K F B 3$ has been shown to be involved in the improvement of insulin sensitivity and protection against inflammation in obese mice [21], indicating a function of PFKFB3 beyond regulation of glycolysis. In addition, we found upregulation of glucose enhancer factor SLC2A4RG, a transcriptional activator of GLUT4 expression [22], in the muscle of diabetic participants after exercise. We did not detect significant differences in GLUT4 mRNA expression before exercise in the two groups, and no increase after exercise, but exercise training interventions in diabetic patients have been shown to result in enhanced protein levels of GLUT4 [23]. It has been reported that the increase in GLUT4 protein is more pronounced in diabetic individuals compared with healthy controls after 6 weeks of exercise training, while the levels were similar at baseline [24]. The increased expression of $S L C 2 A 4 R G$ in diabetic participants in our study may be one possible mechanism. Moreover, our metabolomics data indicate acute improvement in glucose control after a single bout of exercise in diabetic participants. The comparable increase in glucose $R_{\mathrm{a}} / R_{\mathrm{d}}$ during exercise is consistent with earlier reports showing that contraction-induced glucose uptake is not impaired in type 2 diabetes $[9,10,25]$. Acute improvement in glucose control after one single bout of exercise in individuals with impaired glucose tolerance has also recently been shown $[26,27]$.

Metabolite markers reflecting the capability to increase lipolysis and fat oxidation during exercise revealed no impaired regulation in diabetic individuals. Plasma concentrations of acylcarnitines are not only a useful diagnostic marker to detect disorders in mitochondrial fatty acid oxidation but also reflect physiological changes in fatty acid oxidation flux [28-30]. Since the exercise-induced increase in short-, medium- and long-chain acylcarnitines was not different between the control group and the diabetic group, we conclude that the metabolic flexibility of diabetic individuals to enhance fat oxidation during acute exercise is not impaired. A limitation to this interpretation may be that we compared diabetic and NGT participants with similar fitness levels, yet diabetic individuals often have reduced aerobic capacity compared with controls of similar age and weight $[31,32]$. Earlier studies have shown that the oxidation of plasma-derived fatty acids is impaired in diabetes during exercise, while the oxidation of triacylglycerolderived fatty acids is higher $[1,11]$.

In the recovery phase, fatty acids showed a clear groupdependent regulation with constantly higher concentrations in diabetic participants. This could be interpreted as impaired fatty acid oxidation. However, the higher glycerol levels after exercise indicate higher rates of lipolysis and release of fatty 
acids. Moreover, impaired fatty acid uptake in diabetic muscle has been reported to enhance the increase in plasma fatty acids [33]. Importantly, the concentration of plasma fatty acids peaked, together with similar plasma insulin concentrations, in both groups $30 \mathrm{~min}$ post exercise, but defective insulin action in the diabetic participants may blunt the antilipolytic effect of insulin. Studies in mice and humans have shown that the combination of high concentrations of plasma fatty acids and insulin resistance results in incomplete fatty acid oxidation and release of the shortened acyl-CoA as carnitine ester into the circulation $[34,35]$. These shorter acylcarnitines did not accumulate in the diabetic participants in our study; thus, it is unlikely that impaired fat oxidation is the cause of increased plasma fatty acids in the recovery phase.

Our findings reveal a strong association of the lower plasma amino acid levels in the diabetic groups during the trial and differential expression of genes involved in amino acid metabolism in skeletal muscle after exercise (Fig. 4). The lower levels of plasma glycine and glutamine and the higher plasma glutamate levels in the diabetic participants are well in line with those in other reports [36, 37]. The decrease in plasma glycine is possibly due to an increased demand for glutathione in diabetes [38], and this might be amplified by exercise when glutathione synthesis from the amino acid precursors glycine, cysteine and glutamate is enhanced [39]. Compensatory mechanisms to rescue serine, glycine and also creatine levels may be initiated by the differential expression of $P S P H$, GATM and GLDC in the skeletal muscle of diabetic individuals compared with controls. Importantly, a recent report showed increased plasma glycine concentrations after exercise training for 6 months in middle-aged obese individuals at risk of developing diabetes [13]. The increase in glycine in that study was associated with an improvement in insulin sensitivity. Plasma amino acid data, which are available for 75 participants at baseline and after 9 months of combined exercise and diet intervention in the Tübingen lifestyle intervention programme, also showed a slight but significant correlation for increased glycine $\left(p=0.037, R^{2}=0.06\right)$ and serine plasma levels ( $\left.p=0.005, R^{2}=0.10\right)$ with increased insulin sensitivity [40]. Thus, the exercise-induced regulation of genes involved in amino acid synthesis and metabolism in the muscles of the diabetic participants may be important in normalising plasma glycine concentrations but may also be involved in the amelioration of insulin resistance. The lower arginine levels found in diabetic participants may be a potential limitation for the production of nitric oxide during exercise [41]. However, it appeared to be balanced by upregulation of expression of NOS1.

We did not detect elevated levels of branched chain amino acids or of the branched chain amino acid-derived acylcarnitines $\mathrm{C} 3$ and $\mathrm{C} 5$, which have previously not only been associated with diabetes [42, 43] but also with obesity $[35,44]$. It must be considered that the diabetic participants in our study were relatively lean (BMI $24.6 \pm 1.0 \mathrm{~kg} / \mathrm{m}^{2}$ ) and physically fit, which is in contrast to large parts of the diabetic population. Thus, it appears that mechanisms related to altered fat distribution and lipid metabolism are responsible for the increase in branched chain amino acids.

Notably, our exercise study was designed for middle-aged individuals with an ordinary level of physical fitness. In acute exercise tests, intense workloads are sometimes applied to provoke a pronounced molecular response, but the results and the relevance of the identified regulated molecules and pathways might not be transferable to the training sessions performed by the normal population. This could explain why we did not detect an increase in $\beta$-aminoisobutyric acid, which was recently highlighted as a novel $\operatorname{PGC} 1 \alpha$ - and exercise-regulated metabolite involved in protection from metabolic disorders [45].

To conclude, using a complementary metabolomics/ transcriptomics approach we have shown good coherence of differences in plasma metabolite levels in diabetic and NGT participants during exercise, as well as differential expression of muscle genes related to these metabolic pathways. We provide evidence at the molecular level that supports the idea that diabetes does not weaken the metabolic effects of regular physical activity. Diabetic patients appear to derive even greater benefits from moderate exercise, which could provide an efficient means of treating disturbed glucose and amino acid metabolism.

Acknowledgements We thank H. Runge (University Tübingen, Germany) for expert technical assistance.

Funding This work was supported in part by grants from the German Federal Ministry of Education and Research (BMBF) to the German Center for Diabetes Research (DZD e.V.; no. 01GI0925), by grants to the key foundation and creative research group project (nos 21435006 and 21321064 by the Natural Science Foundation of China (NSFC) to GX), the Sino-German Center for Research Promotion (GZ 753 by Deutsche Forschungsgemeinschaft (DFG) and NSFC to GX and RL, and LE 1391/1-1 by DFG to RL), the Leibniz Gemeinschaft (SAWFBN-2013-3 to CW) and the Helmholtz Alliance ICEMED and Helmholtz Portfolio Theme Metabolic Dysfunction and Common Disease (to JB). The Centre of Inflammation and Metabolism (CIM) is supported by a grant from the Danish National Research Foundation (DNRF55). The Centre for Physical Activity Research (CFAS) is supported by a grant from Trygfonden. CIM is part of the UNIK Project: Food, Fitness \& Pharma for Health and Disease, supported by the Danish Ministry of Science, Technology and Innovation. CIM is a member of DD2, the Danish Center for Strategic Research in Type 2 Diabetes (the Danish Council for Strategic Research, grant nos 09-067009 and 09-075724).

Duality of interest The authors declare that there is no potential conflict of interest relevant to this article.

Contribution statement JSH recruited participants, performed preexperimental assessments, designed and performed exercise experiments, and wrote the manuscript. XZ, XL, MH and YL performed metabolomics analyses. MI and MS acquired and analysed data. JB, MHA, HUH, BKP, $\mathrm{RL}$ and GX contributed to the conception and design of the study. RL and 
GX contributed to the analysis and interpretation of the data. PP designed and performed the exercise experiments, interpreted the data and wrote the manuscript. CW designed experiments, interpreted data and wrote the manuscript. All listed authors contributed to drafting the manuscript or revised it critically and approved the final version. $\mathrm{CW}$ is responsible for the integrity of the work as a whole.

\section{References}

1. Kelley DE, Simoneau JA (1994) Impaired free fatty acid utilization by skeletal muscle in non-insulin-dependent diabetes mellitus. $\mathrm{J}$ Clin Invest 94:2349-2356

2. Stefan N, Kantartzis K, Haring HU (2008) Causes and metabolic consequences of fatty liver. Endocr Rev 29:939-960

3. Krogh-Madsen R, Thyfault JP, Broholm C et al (2009) A two-week reduction of ambulatory activity attenuates peripheral insulin sensitivity. J Appl Physiol 108:1034-1040

4. Boule NG, Weisnagel SJ, Lakka TA et al (2005) Effects of exercise training on glucose homeostasis: the HERITAGE Family Study. Diabetes Care 28:108-114

5. Praet SF, van Loon LJ (2008) Exercise: the brittle cornerstone of type 2 diabetes treatment. Diabetologia 51:398-401

6. Hawley JA (2004) Exercise as a therapeutic intervention for the prevention and treatment of insulin resistance. Diabetes Metab Res Rev 20:383-393

7. Turcotte LP, Richter EA, Kiens B (1992) Increased plasma FFA uptake and oxidation during prolonged exercise in trained vs. untrained humans. Am J Physiol 262:E791-E799

8. Hunt K, Wyke S, Gray CM et al (2014) A gender-sensitised weight loss and healthy living programme for overweight and obese men delivered by Scottish Premier League football clubs (FFIT): a pragmatic randomised controlled trial. Lancet 383:1211-1221

9. Wahren J, Hagenfeldt L, Felig P (1975) Splanchnic and leg exchange of glucose, amino acids, and free fatty acids during exercise in diabetes mellitus. J Clin Invest 55:1303-1314

10. Colberg SR, Hagberg JM, McCole SD, Zmuda JM, Thompson PD Kelley DE (1996) Utilization of glycogen but not plasma glucose is reduced in individuals with NIDDM during mild-intensity exercise. J Appl Physiol (1985) 81:2027-2033

11. Blaak EE, Aggel-Leijssen DP, Wagenmakers AJ, Saris WH, van Baak MA (2000) Impaired oxidation of plasma-derived fatty acids in type 2 diabetic subjects during moderate-intensity exercise. Diabetes 49:2102-2107

12. Lewis GD, Farrell L, Wood MJ et al (2010) Metabolic signatures of exercise in human plasma. Sci Transl Med 2:33-37

13. Huffman KM, Slentz CA, Bateman LA et al (2011) Exerciseinduced changes in metabolic intermediates, hormones, and inflammatory markers associated with improvements in insulin sensitivity. Diabetes Care 34:174-176

14. Mahoney DJ, Parise G, Melov S, Safdar A, Tarnopolsky MA (2005) Analysis of global mRNA expression in human skeletal muscle during recovery from endurance exercise. FASEB J 19: $1498-1500$

15. Stepto NK, Coffey VG, Carey AL et al (2009) Global gene expression in skeletal muscle from well-trained strength and endurance athletes. Med Sci Sports Exerc 41:546-565

16. Wolf M, Chen S, Zhao X et al (2013) Production and release of acylcarnitines by primary myotubes reflect the differences in fasting fat oxidation of the donors. J Clin Endocrinol Metab 98:E1137E1142

17. Borno A, Foged L, van Hall G (2014) Glucose and glycerol concentrations and their tracer enrichment measurements using liquid chromatography tandem mass spectrometry. J Mass Spectrom 49: 980-988

18. Wolfe RR, Chinkes DL (2004) Isotope tracers in metabolic research: principles and practice of kinetic analysis. Wiley, New York

19. Rainer J, Sanchez-Cabo F, Stocker G, Sturn A, Trajanoski Z (2006) CARMAweb: comprehensive R- and bioconductor-based web service for microarray data analysis. Nucleic Acids Res 34:W498W503

20. Eichner J, Rosenbaum L, Wrzodek C, Haring HU, Zell A, Lehmann R (2014) Integrated enrichment analysis and pathway-centered visualization of metabolomics, proteomics, transcriptomics, and genomics data by using the InCroMAP software. J Chromatogr B Anal Technol Biomed Life Sci 966:77-82

21. Huo Y, Guo X, Li H et al (2012) Targeted overexpression of inducible 6-phosphofructo-2-kinase in adipose tissue increases fat deposition but protects against diet-induced insulin resistance and inflammatory responses. J Biol Chem 287:21492-21500

22. Knight JB, Eyster CA, Griesel BA, Olson AL (2003) Regulation of the human GLUT4 gene promoter: interaction between a transcriptional activator and myocyte enhancer factor 2A. Proc Natl Acad Sci U S A 100:14725-14730

23. Wang Y, Simar D, Fiatarone Singh MA (2009) Adaptations to exercise training within skeletal muscle in adults with type 2 diabetes or impaired glucose tolerance: a systematic review. Diabetes Metab Res Rev 25:13-40

24. Holten MK, Zacho M, Gaster M, Juel C, Wojtaszewski JF, Dela F (2004) Strength training increases insulin-mediated glucose uptake, GLUT4 content, and insulin signaling in skeletal muscle in patients with type 2 diabetes. Diabetes 53:294-305

25. Martin IK, Katz A, Wahren J (1995) Splanchnic and muscle metabolism during exercise in NIDDM patients. Am J Physiol 269: E583-E590

26. Francois ME, Baldi JC, Manning PJ et al (2014) 'Exercise snacks' before meals: a novel strategy to improve glycaemic control in individuals with insulin resistance. Diabetologia 57:1437-1445

27. Rynders CA, Weltman JY, Jiang B et al (2014) Effects of exercise intensity on postprandial improvement in glucose disposal and insulin sensitivity in prediabetic adults. J Clin Endocrinol Metab 99: 220-228

28. Soeters MR, Sauerwein HP, Duran M et al (2009) Muscle acylcarnitines during short-term fasting in lean healthy men. Clin Sci (Lond) 116:585-592

29. Lehmann R, Zhao X, Weigert $\mathrm{C}$ et al (2010) Medium chain acylcarnitines dominate the metabolite pattern in humans under moderate intensity exercise and support lipid oxidation. PLoS One 5:e11519

30. Hiatt WR, Regensteiner JG, Wolfel EE, Ruff L, Brass EP (1989) Carnitine and acylcarnitine metabolism during exercise in humans. Dependence on skeletal muscle metabolic state. J Clin Invest 84: 1167-1173

31. Bauer TA, Reusch JE, Levi M, Regensteiner JG (2007) Skeletal muscle deoxygenation after the onset of moderate exercise suggests slowed microvascular blood flow kinetics in type 2 diabetes. Diabetes Care 30:2880-2885

32. O'Connor E, Kiely C, O'Shea D, Green S, Egana M (2012) Similar level of impairment in exercise performance and oxygen uptake kinetics in middle-aged men and women with type 2 diabetes. Am J Physiol Regul Integr Comp Physiol 303:R70-R76

33. Corpeleijn E, Saris WH, Blaak EE (2009) Metabolic flexibility in the development of insulin resistance and type 2 diabetes: effects of lifestyle. Obes Rev 10:178-193

34. Koves TR, Ussher JR, Noland RC et al (2008) Mitochondrial overload and incomplete fatty acid oxidation contribute to skeletal muscle insulin resistance. Cell Metab 7:45-56

35. Mihalik SJ, Goodpaster BH, Kelley DE et al (2010) Increased levels of plasma acylcarnitines in obesity and type 2 diabetes and 
identification of a marker of glucolipotoxicity. Obesity (Silver Spring) 18:1695-1700

36. Cheng S, Rhee EP, Larson MG et al (2012) Metabolite profiling identifies pathways associated with metabolic risk in humans. Circulation 125:2222-2231

37. Floegel A, Stefan N, Yu Z et al (2013) Identification of serum metabolites associated with risk of type 2 diabetes using a targeted metabolomic approach. Diabetes 62:639-648

38. Ferrannini E, Natali A, Camastra S et al (2013) Early metabolic markers of the development of dysglycemia and type 2 diabetes and their physiological significance. Diabetes 62:1730-1737

39. Ji LL, Fu R, Mitchell EW (1992) Glutathione and antioxidant enzymes in skeletal muscle: effects of fiber type and exercise intensity. J Appl Physiol (1985) 73:1854-1859

40. Lehmann R, Franken H, Dammeier S et al (2013) Circulating lysophosphatidylcholines are markers of a metabolically benign nonalcoholic fatty liver. Diabetes Care 36:2331-2338
41. Roberts CK, Barnard RJ, Jasman A, Balon TW (1999) Acute exercise increases nitric oxide synthase activity in skeletal muscle. Am J Physiol 277:E390-E394

42. Wang TJ, Larson MG, Vasan RS et al (2011) Metabolite profiles and the risk of developing diabetes. Nat Med 17:448-453

43. Stancakova A, Civelek M, Saleem NK et al (2012) Hyperglycemia and a common variant of GCKR are associated with the levels of eight amino acids in 9,369 Finnish men. Diabetes 61:1895-1902

44. Newgard CB, An J, Bain JR et al (2009) A branched-chain amino acid-related metabolic signature that differentiates obese and lean humans and contributes to insulin resistance. Cell Metab 9:311-326

45. Roberts LD, Bostrom P, O'Sullivan JF et al (2014) $\beta$ Aminoisobutyric acid induces browning of white fat and hepatic $\beta$-oxidation and is inversely correlated with cardiometabolic risk factors. Cell Metab 19:96-108 